\title{
Single-operator cholangioscopy for diagnosis of cholangioadenoma (bile duct adenoma) and its potential impact on surgical management
}

\section{다(1) $(2)$}

\author{
Authors \\ John Eccles ${ }^{1}$, Aducio Thiesen ${ }^{2}$, Gurpal Sandha ${ }^{1}$ \\ Institutions \\ 1 Division of Gastroenterology, University of Alberta \\ Hospital, Edmonton, Alberta, Canada \\ 2 Department of Anatomic Pathology, University of \\ Alberta Hospital, Edmonton, Alberta, Canada
}

submitted 3.5.2018

accepted after revision 31.7 .2018

\author{
Bibliography \\ DOI https://doi.org/10.1055/a-0743-5386 | \\ Endoscopy International Open 2018; 06: E1312-E1316 \\ (c) Georg Thieme Verlag KG Stuttgart · New York \\ ISSN 2364-3722
}

Corresponding author

Dr. Gurpal Sandha, MBBS, FRCPC, Professor of Medicine, University of Alberta, Division of Gastroenterology, University of Alberta Hospital, 2-76 Zeidler-Ledcor Centre, 8540-112 Street, Edmonton, Alberta, Canada, T6G 2X8

Fax: +780-492-1699

gsandha@ualberta.ca

\section{ABSTRACT}

Background and study aims Cholangioadenoma is not recognized commonly and is often only diagnosed on surgical specimens. Direct per oral single-operator cholangioscopy (SOC) allows characterization of common bile duct (CBD) lesions through direct visualization and directed forceps biopsies with potential for impacting surgical management decisions. This is a retrospective review of all SOC cases diagnosed with cholangioadenoma. Patient demographics and outcomes were recorded. Three patients (all male), average age 68 years (range 62-76 years), were identified to have a cholangioadenoma. The clinical indication for SOC was deranged liver enzymes with a dilated CBD and a CBD abnormality identified on biliary imaging. The site of cholangioadenoma was proximal, mid and distal $C B D$, respectively. All patients had a successful SOC with targeted biopsy-proven diagnosis. One patient had a synchronous cholangiocarcinoma and underwent palliative stenting whereas the other two patients underwent appropriate curative resection based on cholangioadenoma location. We conclude that $\mathrm{SOC}$ is safe and effective for diagnosis of cholangioadenoma and has potential impact on decisions for surgical management.

\section{Introduction}

Cholangioadenoma, or bile duct adenoma, is not recognized commonly in clinical practice. It is often only diagnosed on surgical specimens when intervention is undertaken for a presumed malignant biliary lesion [1-9]. Assessment of bile duct lesions represents a common indication for referral for endoscopic retrograde cholangiopancreatography (ERCP). However, differentiating benign from malignant lesions on ERCP is sometimes difficult. With introduction of single-operator cholangioscopy (SOC), it has become possible to characterize common bile duct (CBD) lesions through direct visualization and directed forceps biopsies. This information may further help to guide surgical management. We present our experience with patients identified to have a cholangioadenoma with SOC $(\triangleright$ Table 1 ) and have performed a review of published cases, with specific focus on the role of SOC in the diagnosis and impact on management.

\section{Case reports}

\section{Patient 1}

A 76-year-old man presented with epigastric discomfort, intermittent vomiting and weight loss of $20 \mathrm{lb}$. His liver enzymes were mildly deranged. Abdominal ultrasound showed evidence of biliary dilatation. Computed tomography scan suggested a soft tissue density within the common hepatic duct extending into the right lobe of the liver. There was also a $1-\mathrm{cm}$ stricture in the lower CBD, with wall thickening suspicious for an intraluminal mass. Direct cholangioscopy (SpyGlass, Boston Scientific Corporation, Marlborough, Massachusetts, United States) showed a bulky polypoid lesion at the level of the biliary conflu- 
- Table 1 Demographics and clinical characteristics of patients with cholangioadenoma.

\begin{tabular}{|l|l|l|l|l|}
\hline & CBD site & Clinical features & SOC findings & Treatment \\
\hline Case 1, M 76 years & Proximal & $\begin{array}{l}\text { Abdominal pain with abnormal liver } \\
\text { enzymes and dilated CBD }\end{array}$ & $\begin{array}{l}\text { Synchronous CCA and cholangioadeno- } \\
\text { ma with biopsy confirmation }\end{array}$ & $\begin{array}{l}\text { Palliation with } \\
\text { stenting }\end{array}$ \\
\hline Case 2, M 62 years & Mid & $\begin{array}{l}\text { Abnormal liver enzymes and dilated } \\
\text { CBD }\end{array}$ & $\begin{array}{l}\text { Cholangioadenoma with biopsy confir- } \\
\text { mation }\end{array}$ & Curative resection \\
\hline Case 3, M 65 years & Distal & $\begin{array}{l}\text { Abnormal liver enzymes and dilated } \\
\text { CBD }\end{array}$ & $\begin{array}{l}\text { Cholangioadenoma with biopsy confir- } \\
\text { mation }\end{array}$ & Curative resection \\
\hline
\end{tabular}

CBD, common bile duct; SOC, single-operator cholangioscopy; M, male; CCA, cholangiocarcinoma

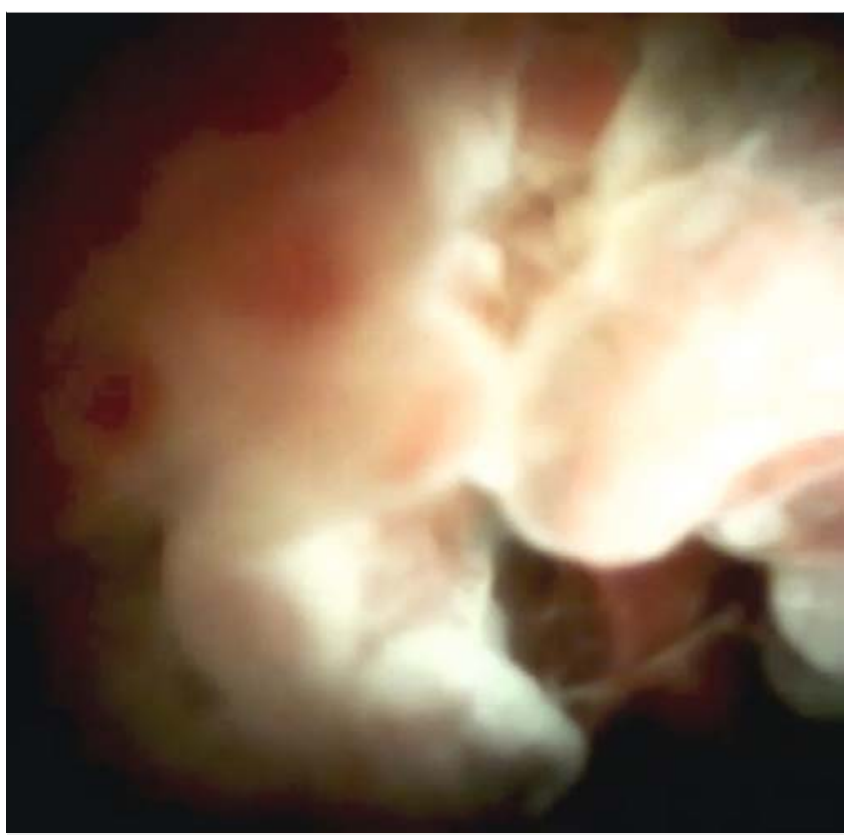

Fig. 1 Papillary fronds within the common hepatic duct seen at direct single-operator per oral cholangioscopy highly suggestive of a cholangioadenoma (imaged with Digital SpyGlass system).

ence extending into the right hepatic duct. This was visually suggestive of a cholangioadenoma ( $\triangleright$ Fig. 1 ). On withdrawal, a circumferential mid CBD lesion with neovascularisation, suspicious for a cholangiocarcinoma, was also seen.

Biopsies were taken with a biopsy forceps from both sites. Cholangiography confirmed the malignant-appearing midCBD stricture and the outline of a soft tissue lesion involving the common hepatic duct extending into the right and left main hepatic ducts correlating with the cholangioadenoma seen on cholangioscopy. Furthermore, the biliary obstruction appeared secondary to the mid-CBD malignant lesion ( $\triangleright$ Fig. 2). A fully-covered self-expanding metal biliary stent was placed across the obstructing mid-CBD stricture. As the proximal lesion was not felt to be causing obstruction at this time, stenting of this area was not performed. Biopsy of the proximal lesion showed only adenomatous epithelium with features felt to be compatible with a cholangioadenoma ( $\triangleright$ Fig.3). Unfortunately, the patient did not qualify for surgery as the

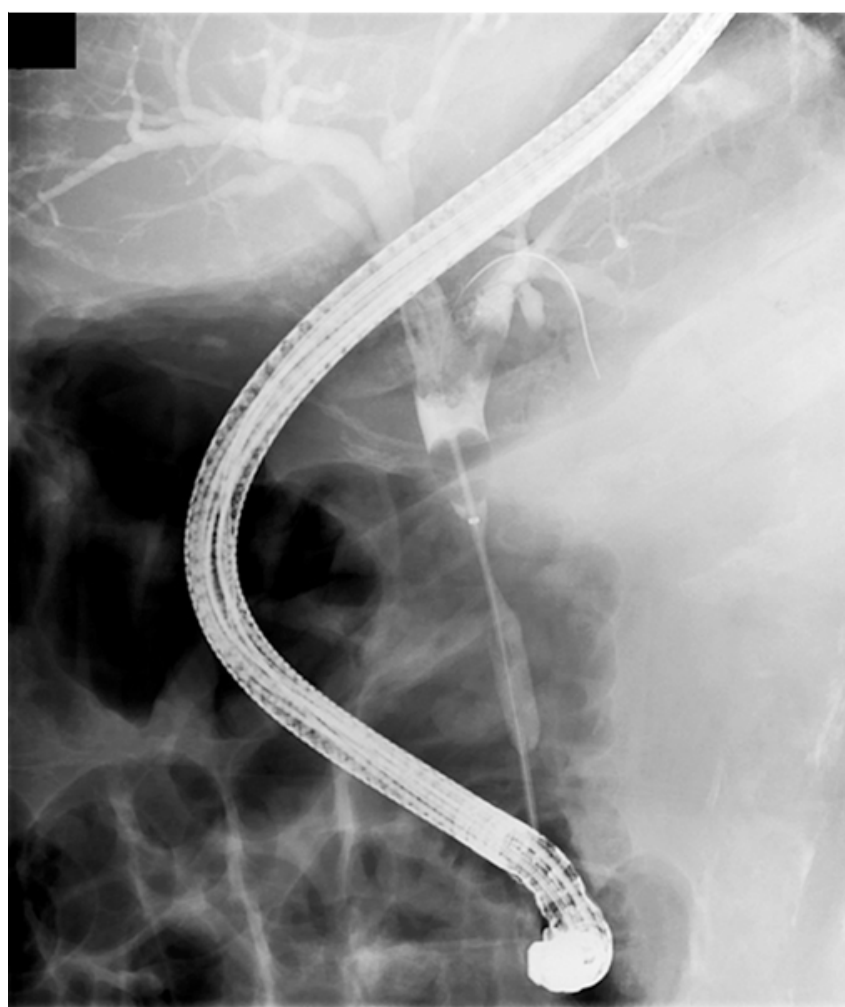

- Fig. 2 Balloon occlusion cholangiography showing synchronous cholangioadenoma at bifurcation (above balloon) and mid-CBD cholangiocarcinoma (below balloon). Occlusion cholangiography facilitated visualization of the cholangioadenoma.

synchronous lesions were not considered amenable to curative resection.

\section{Patient 2}

A 62-year-old man was coincidentally found to have mildly elevated liver enzymes and a dilated CBD on abdominal ultrasound. He was referred for endoscopic ultrasonography, which suggested a non-shadowing intraductal filling defect suggestive of sludge. At ERCP, there was evidence of a mid-CBD intraluminal filling defect with upstream biliary dilatation. After sphincterotomy, a balloon sweep resulted in extraction of sludge but there was persistence of a suspicious mucosal abnormality. Direct cholangioscopy confirmed a sessile polypoid mass within the mid-CBD ( $\triangleright$ Fig. 4). There were no features sus- 


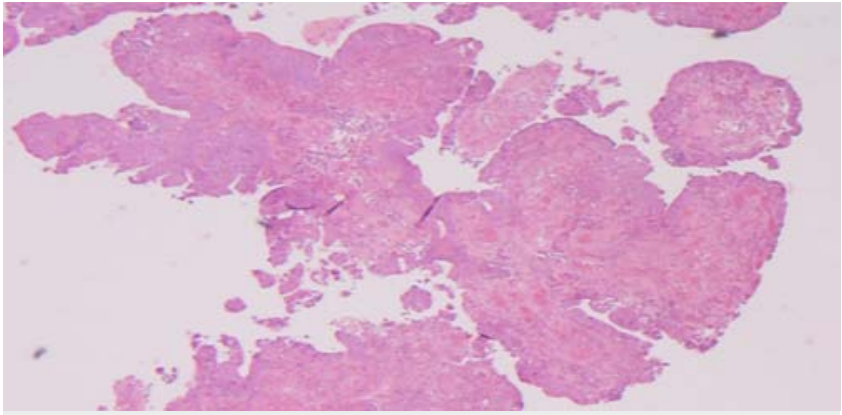

- Fig. 3 Biopsy from common hepatic duct lesion ( $50 \times$ ). Villous architecture lined by hyperchromatic adenomatous epithelium with focal complex architecture in keeping with high grade dysplastic changes.

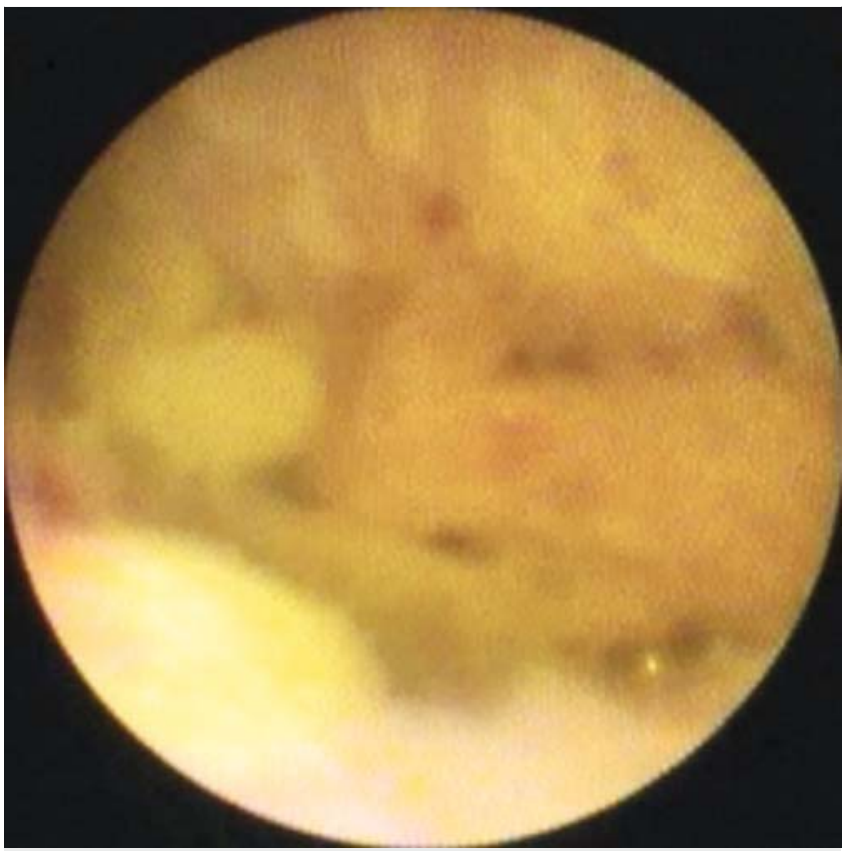

- Fig.4 Papillary fronds within the mid-CBD seen at direct singleoperator cholangioscopy highly suggestive of a cholangioadenoma (imaged with original Legacy SpyGlass system).

picious for malignancy and forceps biopsies (SpyBite, Boston Scientific Corporation, Marlborough, Massachusetts, United States) confirmed an adenoma with focal high-grade dysplasia ( $\triangleright$ Fig.5). The patient proceeded to surgery with curative resection and a roux-en-Y hepaticojejunostomy. Histopathology from the surgical specimen confirmed a bile duct papillary adenoma.

\section{Patient 3}

A 65-year-old man presented with deranged liver enzymes and evidence of CBD dilatation on abdominal CT scan. At ERCP, and following a biliary sphincterotomy, there was evidence of prolapse of a polypoid lesion from the ampulla, felt likely to be in keeping with a cholangioadenoma. The ampulla was normal thereby excluding an ampullary adenoma. On cholangioscopy, the lesion was better characterized and felt to be confined to

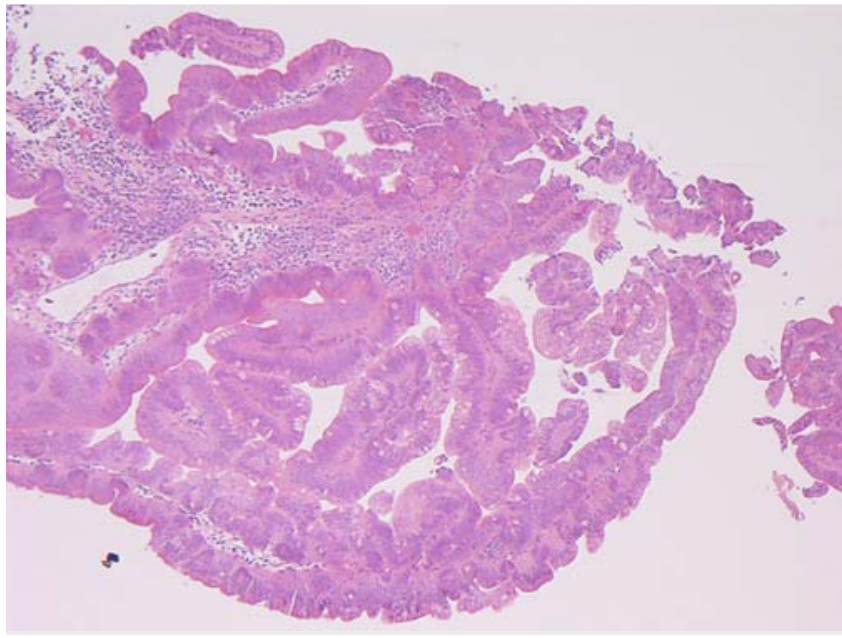

- Fig.5 Biopsy taken from mid-CBD lesion showing superficial hyperchromatic adenomatous epithelium with villous architecture, maintained polarization and no complex architecture, consistent with a cholangioadenoma.

the distal $2 \mathrm{~cm}$ of the common bile duct with no features suggestive of malignancy. A biopsy with regular forceps was obtained and histopathology confirmed adenomatous tissue with no dysplasia. He subsequently underwent curative surgical resection with ampullectomy and reconstruction with both choledochoduodenostomy and pancreaticoduodenostomy. The surgical specimen also confirmed a well-defined villous adenoma with focal high-grade dysplasia.

\section{Discussion}

Cholangioadenoma is described relatively infrequently within literature and rarely seen in clinical practice [1-9]. There have been collectively fewer than 50 cases of cholangioadenoma described in literature. They may be intrahepatic in nature, and detected on cross-sectional imaging or surgical resection, or found within the extra-hepatic biliary tree during investigation of cholestasis. Most published case reports refer to the former type. Chen et al describe their case of a patient found to have an incidental intrahepatic bile duct adenoma on imaging, initially mistaken for hepatocellular carcinoma. In their literature search, they only describe reports of intrahepatic rather than extrahepatic tumors [7]. Kim et al present their case with a tumor at the bifurcation of the common hepatic duct, initially mistaken for a hilar cholangiocarcinoma, with subsequent intrahepatic duct dilatation. Interestingly, their patient underwent a surgical resection and histopathology suggested a tubulovillous adenoma [8]. In their case report, Munshi et al describe a patient presenting with biliary obstruction found to have a small pedunculated polyp prolapsing from the ampulla following biliary sphincterotomy and balloon sweep. Endoscopic resection confirmed this to be an adenoma.

The authors recognize the rare nature of extrahepatic biliary adenomas within the literature. In published case reports, several patients have undergone surgical resection, ranging from 
hepaticojejunostomy to Whipple's procedure depending on the location of the adenoma, to reduce risk of progression to malignancy [5]. Xu et al provide an excellent example of a hilar bile duct adenoma identified on contrast-enhanced transabdominal ultrasonography, ultimately requiring surgical resection due to obstruction. They suggest that the majority of extrahepatic bile duct adenomas are seen distally, particularly at the level of the ampulla, while only about $15 \%$ involve the common hepatic duct and $8 \%$ the hepatic ducts. They also recognize the difficulty in diagnosis using standard imaging techniques given potential to misdiagnose as CBD stones, sludge or even cholangiocarcinoma, helped in their case by using contrast enhancement [3]. Loh et al provide the most extensive and recent literature review on bile duct adenomas as part of their case series published in 2015 [9]. In all three cases in their series, the patients went on to develop malignancy on follow-up of their bile duct adenoma, and they propose the potential for an "aggressive" course with these seemingly benign tumors. They advocate either early surgical intervention or careful surveillance in all cases, supported by the other 36 published cases of bile duct adenoma that they identify within the literature, of which 28 were managed surgically. All the other reports of bile duct adenomas cited in their article are mostly single case-reports. Mavrogenis et al report another rare case of bile duct adenoma diagnosed at direct cholangioscopy. Their patient presented with recurrent cholangitis and was found on "Spyglass" cholangioscopy to have an obstructing adenoma within the right hepatic duct, later treated with endobiliary radiofrequency ablation [6].

Most of the published literature, as evident from the preceding review, is in the form of single case reports, which underscores the uncommon nature of cholangioadenoma. Or perhaps, a lack of appropriate investigative tools may have been the limiting factor as these lesions are not well recognized on cross-sectional imaging (with CT and/or MRCP) and even cholangiography (via ERCP or percutaneous trans-hepatic). Most of these cases have been described on surgical specimens or on cholangioscopy performed via a percutaneous trans-hepatic approach, although the latter approach is an invasive diagnostic approach as even recognized by the authors themselves [10]. There is paucity of literature on the role of direct per oral SOC and as far as we can tell, there has only been one published case report of a cholangioadenoma identified with the currently available SOC system (SpyGlass, Boston Scientific, Marlborough, Massachusetts, United States). Our three cases appear to be the largest reported case-series described using this system.

The role of direct SOC has already been well established in more extensively assessing indeterminate bile duct strictures [11]. Cholangiography, whether performed with an MRCP or $E R C P$, and cross-sectional imaging, may sometimes not be able to differentiate between benign and malignant lesions and even other confounding entities such as sludge and stones. SOC provides a direct assessment of the visual characteristics that are important in differentiating these lesions from one another. In addition, it enables the operator to take biopsies and clarify the extent of involvement within the bile duct. This is invaluable information that can impact the type and extent of surgical intervention in these patients. To date, we have identified three cases of cholangioadenoma diagnosed through direct per oral cholangioscopy. One of our patients had a synchronous mid-CBD cholangiocarcinoma along with a hilar cholangioadenoma as identified at cholangioscopy. The latter lesion was suggested but not clearly discernible on cross-sectional imaging. Both of these lesions were subsequently confirmed on histopathology. Identification of the cholangioadenoma in this patient, in fact, altered surgical management. The other two patients had appropriate curative surgical intervention based on the information, such as location and extent of involvement, obtained with SOC. This reinforces the impact that SOC can have on clarifying characteristics of a cholangioadenoma, differentiating it from CBD stricture mimickers, and then helping guide surgical management in these patients.

Our study certainly has its limitations. It is a single-center retrospective review of SOC cases performed by a single endoscopist. As well, the volume of cases is small although this is still the largest number of cases reported using a per oral SOC system. This underscores the prevalence of this disease although it is certainly possible that with the increasing availability of SOC, there may be more cases of cholangioadenoma identified.

\section{Conclusion}

In conclusion, SOC is safe and effective for diagnosis of cholangioadenoma and has potential impact on decisions for surgical management.

\section{Competing interests}

Dr. Sandha is a consultant for Boston Scientific Corporation and has received honoraria for speaking and proctoring engagements.

\section{References}

[1] Frewin CR, Robertson GS, Roberts-Thomson IC et al. A case of an extrahepatic biliary tract fibro-adenoma in a 35-year-old woman. Aust N Z J Surg 1998; 68: $80-81$

[2] O'Shea M, Fletcher HS, Lara JF. Villous adenoma of the extrahepatic biliary tract: a rare entity. Am Surg 2002; 68: 889-891

[3] Xu H, Chen L. Villous adenoma of extrahepatic bile duct: contrast-enhanced sonography findings. J Clin Ultrasound 2008; 36: 39-41

[4] Sotona O, Cecka F, Neoral C et al. Papillary adenoma of the extrahepatic biliary tract - a rare cause of obstructive jaundice. Acta Gastroenterologica Belgica 2010; 73: $270-273$

[5] Munchi AG, Moustafa AH. Common bile duct adenoma: case report and brief review of the literature. Surg Laparosc Endosc Percutan Tech 2010; 20: e193-194

[6] Mavrogenis G, Deprez PH, Wallon J et al. Bile duct adenoma causing recurrent cholangitis: diagnosis and management with targeted Spyglass access and radiofrequency ablation. Endoscopy 2012; 44: E290 - E291

[7] Chen L, Xu MY, Chen F. Bile duct adenoma: a case report and literature review. World J Surg Oncol 2014; 12: 125 
[8] Kim H], Park EK. A case of bile duct adenoma developed in hepatic hilar portion mimicking Klatskin's tumor. HPB 2016; 18: e527-e528

[9] Loh KP, Nautsch D, Mueller J et al. Adenomas involving the extrahepatic biliary tree are rare but have an aggressive clinical course. Endosc Int Open 2016; 4: E112-E117
[10] Seo DW, Lee SK, Yoo KS et al. Cholangioscopic findings in bile duct tumors. Gastrointest Endosc 2000; 52: 630-634

[11] Chin MW, Byrne MF. Update of cholangioscopy and biliary strictures. World J Gastroenterol 2011; 17: 3864-3869 